Review Article

\title{
Friend or Foe? Essential Roles of Osteoclast in Maintaining Skeletal Health
}

\author{
Haixing Wang $\mathbb{D}^{1,2}$ Guangpu Yang, ${ }^{1}$ Yinbo Xiao, ${ }^{3}$ Guotian Luo $\mathbb{D}^{4,5}$ Gang Li ${ }^{4},{ }^{1,2}$ \\ and Ziqing $\operatorname{Li} \mathbb{B}^{6,7}$ \\ ${ }^{1}$ Department of Orthopaedics \& Traumatology, Faculty of Medicine, The Chinese University of Hong Kong, \\ Prince of Wales Hospital, Hong Kong, China \\ ${ }^{2}$ Stem Cells and Regenerative Medicine Laboratory, Li Ka Shing Institute of Health Sciences, \\ The Chinese University of Hong Kong, Prince of Wales Hospital, Hong Kong, China \\ ${ }^{3}$ Centre for the Cellular Microenvironment, Institute of Molecular, Cell \& Systems Biology, \\ College of Medical, Veterinary and Life Sciences, University of Glasgow, Glasgow, UK \\ ${ }^{4}$ Université de Paris, CNRS, INSERM, B3OA, Paris, France \\ ${ }^{5}$ Ecole Nationale Vétérinaire d'Alfort, B3OA, Maisons-Alfort, France \\ ${ }^{6}$ Department of Anatomy and Cell Biology, School of Dental Medicine, University of Pennsylvania, Philadelphia, PA, USA \\ ${ }^{7}$ Department of Basic and Translational Sciences, School of Dental Medicine, University of Pennsylvania, Philadelphia, PA, USA
}

Correspondence should be addressed to Ziqing Li; ziqingli@upenn.edu

Received 26 October 2019; Accepted 27 January 2020; Published 4 March 2020

Academic Editor: Heather F. Smith

Copyright $(92020$ Haixing Wang et al. This is an open access article distributed under the Creative Commons Attribution License, which permits unrestricted use, distribution, and reproduction in any medium, provided the original work is properly cited.

Heightened activity of osteoclast is considered to be the culprit in breaking the balance during bone remodeling in pathological conditions, such as osteoporosis. As a "foe" of skeletal health, many antiosteoporosis therapies aim to inhibit osteoclastogenesis. However, bone remodeling is a dynamic process that requires the subtle coordination of osteoclasts and osteoblasts. Severe suppression of osteoclast differentiation will impair bone formation because of the coupling effect. Thus, understanding the complex roles of osteoclast in maintaining proper bone remodeling is highly warranted to develop better management of osteoporosis. This review aimed to determine the varied roles of osteoclasts in maintaining skeletal health and to highlight the positive roles of osteoclasts in maintaining normal bone remodeling. Generally, osteoclasts interact with osteocytes to initiate targeted bone remodeling and have crosstalk with mesenchymal stem cells and osteoblasts via secreted factors or cell-cell contact to promote bone formation. We believe that a better outcome of bone remodeling disorders will be achieved when proper strategies are made to coordinate osteoclasts and osteoblasts in managing such disorders.

\section{Introduction}

Bone is a dynamic organ that continuously remodels in a well-orchestrated manner to support body-required mechanical characteristics and maintain calcium homeostasis throughout one's lifetime $[1,2]$. This constant remodeling process requires delicate coordination from multiple cell types, in which hematopoietic stem cell- (HSC-) derived osteoclast (OC) lineage and bone marrow mesenchymal stem cell- (BMSC-) derived osteoblast (OB) lineage receive the most attention [3-5]. Balance between bone resorption by OCs and bone formation by OBs is usually maintained during the physiological process but dies away under pathological conditions, such as inflammation, diabetes, aging, and cancer, resulting in bone remodeling-related disorders and diseases, such as osteoporosis, periodontitis, inflammatory arthritis, Paget's disease, or tumor-induced osteolytic bone metastasis [6-10]. OCs, the giant cells that are responsible for bone removal in the skeletal family, have always been considered to be the main culprit in these disorders and diseases because of its overactive functionalities under pathological conditions [7, 8]. Therefore, 
antiresorptive drugs, such as bisphosphonates, receptor activator of nuclear factor- $\kappa \mathrm{B}$ (RANK) ligand (RANKL) inhibitor, estrogen, or selective estrogen receptor modulators, are prevalent therapeutics that target osteolysis and rescue bone loss [11-13].

Recently, with the in-depth study in bone physiology, OCs, the giant (but not a fool), are manifesting more complex identities beyond their resorptive function. In particular, the reciprocal interactions between bone cells are attracting much attentions [14-16], because of the advanced understanding of the bone coupling between osteoclastic bone resorption and osteoblastic bone formation $[3,17,18]$. Through cell-cell contact, cell-bone matrix interaction, and paracrine factors, OCs have crosstalk with other bone cells, stem cells, and immune cells in the bone microenvironment, which affects recruitment, differentiation, and function of not only themselves but also the other cells [19-21]. This effect of OCs on other cells is more apparent during skeletal aging due to deteriorations on mesenchymal stem cell/ mesenchymal stromal cell- (MSC-) derived osteogenesis and chondrogenesis, while HSC-derived osteoclastogenesis advances with increasing age, thereby gaining the initiative in the bone remodeling process and functioning predominantly over other factors [22-25]. It should be noted that OC-derived activities have both positive and negative effects, and those "pure" antiresorptive drugs (bisphosphonates or denosumab) for age-related bone disorder usually inhibit bone resorption with a concomitant reduction in bone formation owing to bone coupling, indicating the importance of OCs in maintaining normal bone remodeling after adulthood [11, 26, 27].

This review aimed to determine the essential roles of OC not just as a bone eater during bone remodeling but also as a positive contributor to the bone microenvironment and skeletal health. Specifically, we discuss how OCs contribute to the recruitment and differentiation of MSCs, as well as the following bone formation during remodeling. We hope this review can provide a different perspective on recognizing OCs when strategies are created to develop ideal therapeutic agents that target bone remodeling disorders characterized by excessive OC activity.

\section{Osteoclasts and Bone Remodeling}

Unlike bone modeling, which does not require coupled activities of OCs and OBs during skeletal growth and development, bone remodeling demands anatomically or spatially coupled activities of OCs and OBs to replace the old and damaged bone and to maintain calcium homeostasis in the body throughout one's life [28]. Each year, approximately 3 to 4 million basic multicellular units (BMUs) responsible for bone remodeling are initiated, and about 1 million of them are highly active as a standby for participating in bone turnover in the adult skeleton [28-30]. The remodeling process inside the BMUs does not occur randomly along the bone surface, but rather at specific sites, and it follows a well-orchestrated sequence of events that are typically divided into five stages: the activation of OC recruitment, initiation of osteoclastic bone resorption, transition from catabolism to anabolism due to OC apoptosis and $\mathrm{OB}$ recruitment, formation of the new organic matrix by OBs, and subsequent mineralization over time $[28,31]$. In healthy adults, under physiological conditions, bone mass can be stable for one or two decades after reaching the peak volume due to a balance of the bone resorption and bone formation, that is, until age-related imbalance starts (heightened OC activity and reduced OB performance) $[6,22,28]$.

OCs, the unique bone-resorbing cells, arise from HSCs and belong to the monocytic family [21, 32]. In the activation phase of bone remodeling, mononuclear OC precursors in the bone marrow or from blood circulation are attracted to prospective resorption sites, where they attach to the matrix surface and further differentiate into mature OCs (giant multinucleated cells) via cell fusion, termed as "multinucleation" [5, 32-34]. Mature OCs start to generate sealing zones on the targeted matrix surface during the resorption phase via the rearrangement of the cytoskeleton and the formation of a dense belt-like structure called the "actin ring" $[35,36]$. The actin ring encloses the plasma membrane and makes it into a highly convoluted ruffled border which then serves as an exit site for protons and lysosomal proteases, such as cathepsin K (CTSK) to be secreted into the resorption lacunae, facilitating hydrolyzation and solubilization of the inorganic and organic components of bone $[5,20,37]$. By sensing the concentration of extracellular calcium $\left[\mathrm{Ca}^{2+}\right]_{0}$ around the cell and responding to the change of intracellular $\mathrm{Ca}^{2+}$ concentration $\left[\mathrm{Ca}^{2+}\right]_{\mathrm{i}}$, OCs switch between the resorbing state featured by possessing actin rings and the nonresorbing/migrating state featured by scattered podosomes [38-40]. The resorbing activity of OCs gradually declines when basal $\left[\mathrm{Ca}^{2+}\right]_{i}$ increases, whereas lower $\left[\mathrm{Ca}^{2+}\right]_{\mathrm{i}}$ reduces cell motility but enhances the anchoring capacity of the cell onto the bone matrix surface $[38,41]$. Once resorption at one site is completed, OCs can move and start a new resorption cycle somewhere else or undergo apoptosis based on their lifespan [32, 36]. Among key molecules and signaling pathways involved in the process of osteoclastogenesis and resorption activity, RANK signaling is dominant through the entire life cycle of OCs and can be further amplified by costimulatory signals from immunoreceptor tyrosine-based activation motif- (ITAM-) associated immunoglobulin-like receptor (IgLR) signaling [38, 42-44]. Details of the RANK signaling network, along with other critical pathways that cooperate with it, such as calcium signaling pathway $\left(\mathrm{Ca}^{2+} /\right.$ calmodulin/calcineurin/ NFATc1) and oxidative stress response pathway (ROS/Nrf2/ Keap1), have been well summarized in several excellent papers and will not be discussed further in this review [32, 38, 45-47].

Recent advances widely explored the origins of OCs and associated them with aging and other pathological scenarios. It was not until the last decade that researchers started to decipher how aging affects the skeletal system tremendously. While osteogenic and chondrogenic differentiation from MSCs deteriorates, aging upgrades OC progenitors in both quality and quantity, including increased intrinsic expression of c-Fms and RANK, and enlarged OC progenitor pool 
[22-25]. As the origin of the OC progenitor, HSCs contribute to the reinforcement of the progenitors' pool by giving a bias toward myeloid development over lymphoid differentiation with increasing age [23, 48]. Madel et al. recently summarized different origins of OCs in an agedependent manner (Figure 1): the embryonic erythro-myeloid progenitor (EMP) lineage during the embryonic and postnatal period, bone marrow myeloid/monocyte/macrophage (BMMs) lineage during adulthood, and conventional/ mature monocytes (MNs), as well as dendritic cells (DCs) under inflammatory conditions which are usually seen in old age $[21,49]$. In addition to the promotion of OC progenitors during aging, OC supporting cells, such as OBs, B cells, and $\mathrm{T}$ cells, also contribute to osteoclastogenesis by increasing RANKL expression and reducing osteoprotegerin (OPG) level in the bone microenvironment, although the population of these cells decreases with increasing age $[24,50-52]$. Therefore, OC is vulnerable to be treated as a "foe" of skeletal health because of hyperactivity, especially in aged individuals. However, it does not negate its substantial role as a "friend" in removing the old and damaged bone, as well as a positive contributor during bone formation after adulthood, which has become more understandable in the last few years.

Several in vitro studies indicated that OC-derived factors directly affect MSC recruitment and OB differentiation [53-56]. Karsdal et al. reported that conditioned media (CM) from human OCs increased bone nodule formation in a dose-dependent manner, which was further confirmed by Kreja et al. [53, 54, 56]. Interestingly, they also found that the effect of OCs on MSC migration and OB differentiation can be independent of their resorption activity. Likewise, Henriksen's study indicated that mature OCs were sources of anabolic stimuli for OBs, and their interaction with the matrix can strongly affect the anabolic signals from OCs to OBs [55]. Conversely, a reduced number of OBs and bone formation were found in OC-poor osteopetrosis, indicating a critical role of OCs in regulating bone anabolic function [57]. All these findings suggest complex identities of the giant beyond the resorption function.

\section{Osteoclasts and the Initiation of Bone Remodeling}

The initiation phase of bone remodeling includes the recruitment of OC precursors, differentiation and functioning of OCs, and maintenance of bone resorption [28, 31]. The initiation of osteoclastogenesis largely depends on the crosstalk between OC precursors and the OB lineage cells. Emerging data supports the central regulatory role of osteocytes in the initial stage of bone remodeling [58-62]. As the most abundant cells in bone that are derived from OBs and embedded in the bone matrix, osteocytes play a role in determining which bone surface OCs are about to resorb $[58,59]$. Through a network of osteocyte canaliculi, osteocytes can detect microfractures and microcracks in bone and contact other cells, such as OBs, on the bone surface. Bone fatigue induces apoptosis of osteocytes, which are localized to regions that contain microcracks, and this apoptosis was observed to precede OC invasion in the damaged area, which triggers subsequent bone remodeling in the targeted region [63].

Osteocytes have also crosstalk with OCs via secreted proteins. Osteocytes can control OC function by secreting RANKL and transforming growth factor beta (TGF- $\beta$ ) $[64,65]$. RANKL, one of the essential osteoclastogenic factors, is mainly secreted by osteocytes [65-67]. Nakashima et al. [65] demonstrated that osteocytes express a much higher amount of RANKL and have a better capacity to support osteoclastogenesis than OBs and bone marrow stromal cells, which is a strong evidence for the crosstalk between osteocytes and OCs in bone remodeling. The MLO-Y4 osteocyte-like cell line represents a good model for studying the soluble interactions between osteocytes and OCs [64]. When mechanical scratching was applied to MLO-Y4 cells, enhanced secretion of osteoclastogenic factors, RANKL, and the monocyte colony-stimulating factor (M-CSF) was observed. The mechanical scratching of osteocytes induced the formation of tartrate-resistant acid phosphatase- (TRACP-) positive cells on top of the gel along the damaged region. No TRACP-positive cells were formed in the peripheral regions [59]. These findings indicate that soluble factors secreted from damaged osteocytes could locally induce and activate the initial phase of OCs formation.

The initiation of bone remodeling at the targeted bone site is essential for the renewal of an old or damaged bone matrix to prevent the skeleton from aging. Failure to trigger bone remodeling can result in accumulated microdamage and hypermineralization, which leads to reduced bone quality and increased fracture risk. Thus, retaining the crosstalk between OCs and osteocytes is beneficial for skeletal health when managing high turnover bone disorders, such as osteoporosis.

\section{Effect of Osteoclasts on Mesenchymal Stem Cell Recruitment and Osteoblast Differentiation}

After the old or damaged bone is resorbed by OCs, bone remodeling enters the second phase: the transition of $\mathrm{OC}$ to $\mathrm{OB}$ activity. In this reversal phase of bone remodeling, the microenvironment created by OC activity provides signals that aid in the cessation of bone resorption and the initiation of bone formation via the recruitment and differentiation of MSCs $[17,68]$. The bone resorptive microenvironment is built by multiple factors that are released from the bone matrix during bone resorption or directly secreted by OCs locally, which also contribute to the establishment of the osteogenic microenvironment that promotes the recruitment of MSCs [4, 69-71]. MSCs are multipotent stem cells that are capable of differentiating into various cell types, such as OBs, adipocytes, and chondroblasts $[72,73]$. In the bone marrow, MSCs are located around sinusoids and the perivascular network in the stroma [74, 75]. During bone remodeling and fractured-bone regeneration, MSCs migrate to the bone surface or fracture site and then differentiate into OBs to reconstruct the bone [76], subsequent to the 


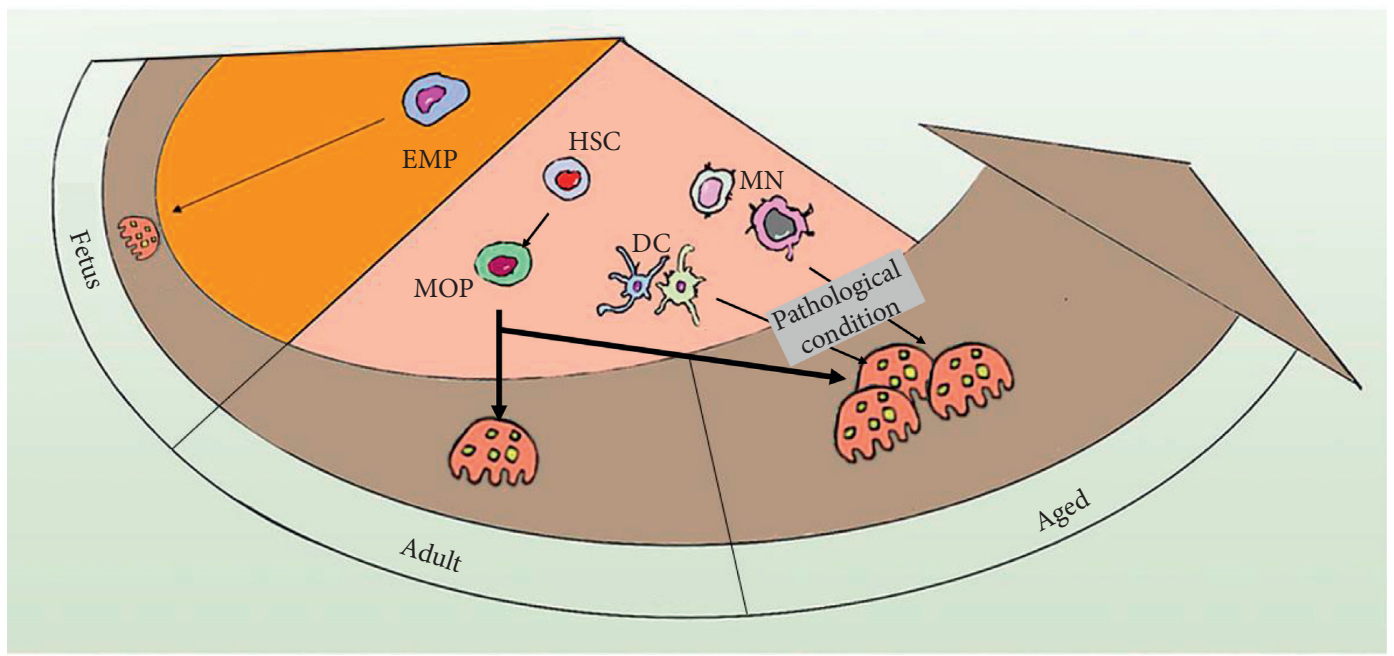

FIGURE 1: Origins of osteoclasts in an age-dependent manner [21]. Osteoclasts (OCs) differentiate from the embryonic erythro-myeloid progenitor (EMP) lineage during the embryonic and postnatal period. In adulthood, bone marrow myeloid/monocyte/macrophages (BMMs) derived from hematopoietic stem cells (HSCs) are the main origin of osteoclasts. Moreover, monocytes (MNs) and dendritic cells (DCs) are also important origins of osteoclasts in aged or pathological conditions. MOP: macrophage/osteoclast progenitor.

osteoclastic resorptive phase. It has been well demonstrated that local growth factors and signals play important roles in the recruitment and commitment of MSCs [77], such as the bone morphogenetic protein (BMP) family [78], insulin-like growth factor (IGF) $[79,80]$, TGF- $\beta[68,81]$, fibroblast growth factor 2 (FGF-2) [82], vascular endothelial growth factors (VEGF) [78], and platelet-derived growth factors (PDGFs) [83, 84]. Moreover, emerging evidence showed that many of these local factors are associated with the viability and activity of OCs $[17,20,54]$.

\subsection{Osteoclastic Resorption Releases Bone Matrix Embedded} Factors and Recruits Mesenchymal Stem Cells. Factors released from the bone matrix during bone resorption may be the first signal from OCs that has been found to affect MSCs. The bone matrix contains many latent growth factors that are deposited by OBs during matrix construction and then released by osteoclastic resorption on the bone surface $[85,86]$. Howard et al. [87] firstly proposed that the release of coupling factors embedded in the bone matrix may positively affect MSC-derived osteogenesis. To date, several matrix-derived factors have been identified as potential factors involved in bone remodeling, such as TGF- $\beta$ [85, 88], IGF-1 [69], bone morphogenetic protein (BMP)-2 [89, 90], and vascular endothelial growth factor (VEGF) [91]. In particular, matrix-derived TGF- $\beta 1$ and IGF-1 have shown definite effects linking bone resorption to MSC recruitment and differentiation based on genetically manipulated mice data. Tang et al. [88] demonstrated that TGF- $\beta 1$ released during OCs culture on bone slices in vitro induces the migration of MSCs. They also found high levels of active TGF- $\beta 1$ in the bone resorptionconditioned media (BRCM) when functional OCs were cultured with bone slices in vitro, whereas active TGF- $\beta 1$ was barely detectable in the conditioned media prepared without bone slices. Moreover, BRCM prepared using OCs generated from normal mice and bone slices prepared from TGF- $\beta 11$ knockout (TGF- $\beta 1-/-$ ) mice was significantly less effective in promoting the migration of BMSCs [88], demonstrating that matrix-derived TGF- $\beta 1$ plays a key role in recruiting MSCs. Similarly, it has also been well demonstrated that IGF-1 released from the bone matrix by functioning OCs stimulated OB differentiation of MSCs by activating the mammalian target of rapamycin (mTOR) through the PI3K-Akt pathway [69].

4.2. Osteoclast-Secreted Factors Recruit Mesenchymal Stem Cells and Promote Osteoblast Differentiation. Besides the matrix-derived factors, increasing data also suggest that factors directly secreted by OC lineage cells play a crucial role in coupling osteoclastic bone resorption with osteoblastic bone formation. Henriksen et al. [55] performed a research to address the anabolic effect of OC linage cells in different stages. They collected the conditioned medium (CM) from macrophages, pre-OCs, and mature functional or nonresorbing OCs and tested their effects on osteogenesis in vitro. Their results suggested that $\mathrm{CM}$ from macrophages did not induce bone formation, while CM from mature OCs promoted osteogenesis, both dependent on and independent of their resorptive activity. Kim et al. [56] also conducted a research to explore when the coupling factors are taking effect during osteoclastogenesis. They found that CM from OCs in the early stage of differentiation predominantly enhanced the migration of osteoblastic lineage cells, confirming that OCs play an important role in the coupling by stimulating pre-OBs migration.

To date, increasing studies have identified numerous secreted molecules from OCs and explored their potential roles in bone remodeling. In Table 1, we have summarized the OC-secreted factors and their effects on MSC migration, $\mathrm{OB}$ differentiation in vitro, or bone formation in vivo. Among them, factors including Afamin [56], CXCL16 [98], 
TABLE 1: Summary of osteoclast-secreted factors on bone remodeling.

\begin{tabular}{|c|c|c|c|}
\hline \multicolumn{3}{|c|}{ Factor secreted by osteoclasts $\quad$ Effect on bone remodeling } & Reference \\
\hline \\
\hline Afamin & Afamin & $\begin{array}{c}\text { Afamin secreted by osteoclasts in the early stage of differentiation } \\
\text { stimulates preosteoblasts migration in vitro via the Akt-signaling } \\
\text { pathway } \\
\text { Afamin can prevent Wnt proteins from aggregating and deliver Wnt } \\
\text { ligands to its receptors on the cell surface, which plays an important role } \\
\text { in osteogenesis }\end{array}$ & {$[56,92]$} \\
\hline BMP6 & Bone morphogenic protein 6 & $\begin{array}{c}\text { Synthesis of BMPs has been confirmed in osteoclasts using } \\
\text { immunocytochemistry and in situ hybridization } \\
\text { BMP6 promotes osteoblast differentiation }\end{array}$ & {$[93,94]$} \\
\hline $\mathrm{C} 3 \mathrm{a}$ & Complement component $3 \mathrm{a}$ & $\begin{array}{l}\text { C3 gene expression increases during osteoclastogenesis, and the cleavage } \\
\text { product C3a is detected in the conditioned medium of osteoclasts } \\
\text { C3a promotes osteoblast differentiation }\end{array}$ & [95] \\
\hline CT-1 & Cardiotrophin-1 & $\begin{array}{l}\text { CT-1 promotes osteoblast differentiation } \\
\text { Neonatal Ct-1-/- mice have decreased osteoblast numbers and BV/TV }\end{array}$ & [96] \\
\hline CTHCR1 & $\begin{array}{l}\text { Collagen triple repeat } \\
\text { containing } 1\end{array}$ & $\begin{array}{c}\text { CTHCR1 is secreted by mature bone-resorbing osteoclasts } \\
\text { CTHCR1 stimulates osteoblast differentiation } \\
\text { Osteoclast-specific deletion of CTHCR1 in mice resulted in osteopenia } \\
\text { due to reduced bone formation }\end{array}$ & [97] \\
\hline CXCL16 & $\begin{array}{l}\text { Chemokine }(\mathrm{C}-\mathrm{X}-\mathrm{C} \text { motif }) \\
\text { ligand } 16\end{array}$ & $\begin{array}{l}\text { TGF- } \beta 1 \text { released from the bone matrix during bone resorption induces } \\
\text { CXCL16 production in osteoclasts, which promotes migration of } \\
\text { osteoblast progenitors in bone remodeling }\end{array}$ & {$[98]$} \\
\hline HGF & Hepatocyte growth factor & $\begin{array}{l}\text { Osteoclasts can synthesize and secrete biologically active HGF, which } \\
\text { promotes osteoblast proliferation and increases osteopontin expression } \\
\text { in osteoblasts }\end{array}$ & {$[99,100]$} \\
\hline PDGF-BB & $\begin{array}{l}\text { Platelet-derived growth factor } \\
\qquad \text { BB }\end{array}$ & $\begin{array}{c}\text { PDGF-BB induces MSC migration, but it inhibits osteoblast } \\
\text { differentiation }\end{array}$ & {$[53,101-103]$} \\
\hline S1P & Sphingosine-1-phosphate & $\begin{array}{l}\text { S1P stimulates MSC migration and promotes osteoblast differentiation } \\
\text { Raising S1P levels in adult mice markedly increased bone formation }\end{array}$ & [104-106] \\
\hline SLIT3 & slit guidance ligand 3 & $\begin{array}{l}\text { Osteoclast-secreted SLIT3 synchronously inhibits bone resorption and } \\
\text { stimulates bone formation } \\
\text { SLIT3 injection in mice markedly rescued bone loss after ovariectomy } \\
\text { surgery }\end{array}$ & {$[107]$} \\
\hline TRAP & $\begin{array}{l}\text { Tartrate-resistant acid } \\
\text { phosphatase }\end{array}$ & $\begin{array}{c}\text { TRAP promotes osteoblast differentiation } \\
\text { TRAP overexpressing transgenic mice have an increased rate of bone } \\
\text { turnover }\end{array}$ & {$[108,109]$} \\
\hline $\begin{array}{l}\text { Vesicular } \\
\text { RANK }\end{array}$ & $\begin{array}{l}\text { Vesicular TNF receptor } \\
\text { superfamily member } 11 \mathrm{~A}\end{array}$ & $\begin{array}{l}\text { Mature OCs secrete vesicular RANK, which binds osteoblastic RANKL } \\
\text { and promotes bone formation via triggering RANKL reverse signaling }\end{array}$ & {$[110]$} \\
\hline Wnt10b & Wnt family member $10 \mathrm{~b}$ & $\begin{array}{l}\text { Wnt10b expression increases during osteoclastogenesis } \\
\text { Wnt10b promotes mineralization }\end{array}$ & {$[104]$} \\
\hline \multicolumn{4}{|c|}{ Osteoclast-derived inhibiting factors of bone formation } \\
\hline LIF & Leukemia inhibitor factor & $\begin{array}{l}\text { LIF inhibits TGFb1-induced osteoblast migration } \\
\text { Sema4D suppresses bone formation by inhibiting IGF-1 signaling }\end{array}$ & [98] \\
\hline Sema4D & Semaphorin 4D & $\begin{array}{c}\text { Sema4d-/- mice show an osteosclerotic phenotype due to augmented } \\
\text { bone formation }\end{array}$ & [111] \\
\hline SOST & Sclerostin & $\begin{array}{l}\text { SOST is expressed in osteoclasts from aged mice and inhibits osteoclast- } \\
\text { mediated stimulation of mineralization }\end{array}$ & [112] \\
\hline $\begin{array}{l}\text { Exosomal miR- } \\
214-3 p\end{array}$ & Exosomal miR-214-3p & $\begin{array}{l}\text { miR-214-3p reduces bone formation in elderly women with fractures and } \\
\text { in ovariectomized mice }\end{array}$ & {$[113,114]$} \\
\hline
\end{tabular}

PDGF-BB [101, 102], and S1P [104, 105] secreted by OCs can promote the migration of MSC or OB progenitors, and factors such as BMP6 [98], C3a [95], CT-1 [96], CTHCR1 [97], HGF [99, 100], SLIT3 [107], Trap [108, 109], and vesicular RANK [110] exhibit enhancing effects on $\mathrm{OB}$ differentiation in vitro or bone formation in vivo. However, some other factors such as Sema4D [111], sclerostin [112], and exosomal miR-214-3p [113] show an inhibiting effect on bone formation. These factors may act as a "fine-tuning" mediator of the bone remodeling process in the BMUs, by inhibiting the remodeling process under some special conditions. Besides, these factors are often highly expressed in OCs from aged or ovariectomized mice, suggesting that they may play a role in bone remodeling disorders during aging. Overall, on the basis of the current findings, most OC-secreted factors show enhancing effects on MSC recruitment or OB differentiation, indicating an essential role of OCs in maintaining normal bone formation during the remodeling process. 


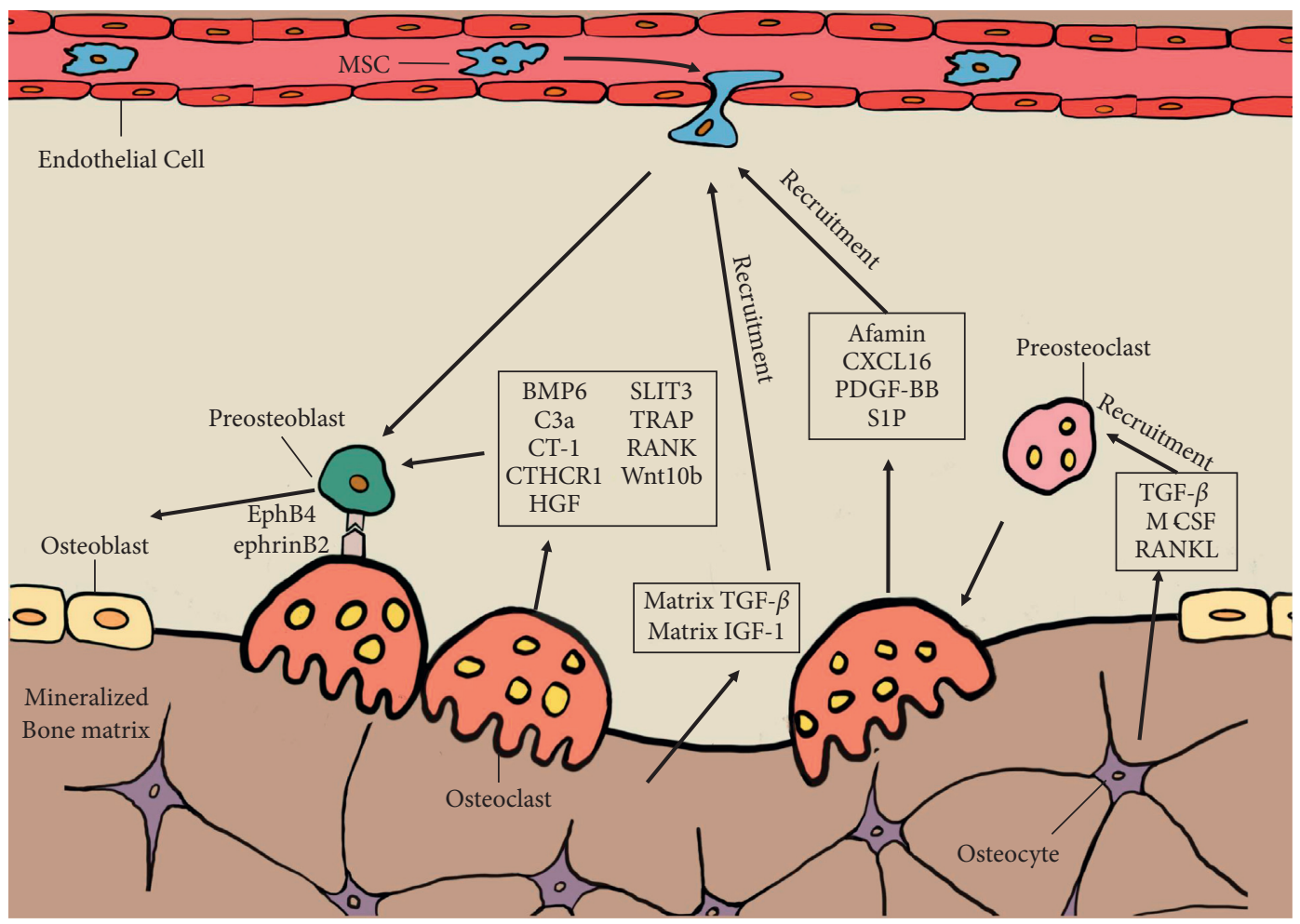

FIGURE 2: Schematic illustration of the interaction between osteoclast (OC) and osteoblast (OB) lineage cells in bone remodeling. OC precursors are activated by TGF- $\beta$, M-CSF, and RANKL secreted by osteocytes and attracted to prospective resorption sites. Once attached to the bone matrix, OC precursors can differentiate into mature OCs. Mature OCs will further acidify and resorb the mineralized bone matrix by pumping hydrogen ions into resorptive captivity through their ruffled border structure. During bone resorption, OC can release several coupling factors, such as matrix-derived TGF- $\beta$, matrix-derived IGF-1, Afamin, CXCL16, PDGF-BB, and S1P et al., which recruit circulated mesenchymal stem cells (MSCs) to the resorption area. Besides, OC also secretes some other coupling factors, such as BMP6, SLIT3, C3a, TRAP, CT-1, and RANK et al., which further promote the differentiation from MSCs towards OBs. Additionally, the ephrinB2/ ephB4 interaction between $\mathrm{OC}$ and $\mathrm{OB}$ precursors suppresses the bone resorption activity of OCs, whereas such interaction could trigger OB differentiation of $\mathrm{OB}$ precursors and enhance bone formation.

\subsection{Osteoclast and Osteoblast Cell-Cell Contact: A Potential} Mechanism of Transition in Bone Remodeling. OCs and OB lineage cells can also communicate through cell-cell contact to achieve the coupling of bone resorption and formation. Traditionally, it has been thought that OCs and OBs do not occur simultaneously at the same BMUs, and direct contact between mature OBs and functioning OCs is relatively rare [115]. In recent years, direct OC-OB contact in vivo has been detected using transmission electron microscopy [31] and intravital two-photon imaging [116]. Furuya et al. demonstrated that mature OCs became nonresorptive when they made contact with mature OBs, and intermittent administration of the parathyroid hormone (PTH) led to an increase in cell-cell contact between OCs and OBs, which causes bone anabolic effects [116].

How does the cell-cell contact cause bidirectional effects between OCs and OBs? EphrinB2/EphB4 interaction between OCs and OBs plays a role in the transition from bone resorption to the formation. Ephrin/Eph family members are local mediators of cell function through contact-dependent manner during various developmental processes $[117,118]$. Interaction between ephrin-expressing and Ephexpressing cells leads to bidirectional signal transduction. Mature OCs express ephrinB2, whereas OB precursors express EphB4 (Figure 2). Forward signaling through the EphB4 receptor into OB precursors enhances osteogenic differentiation by reducing RhoA activity, while reverse signaling through ephrinB2 ligand into OCs suppresses OC function by inhibiting the osteoclastogenic c-Fos-NFATc1 cascade [119]. However, it has also been suggested that mice lacking ephrinB2 showed no skeletal abnormalities [119]. Thus, the role of ephrinB2/EphB4 interaction between OCs and $\mathrm{OBs}$ in the transition from bone resorption to formation needs further confirmation.

\section{Summary and Perspectives}

The skeletal system provides mechanical support, protects vital organs, and controls mineral homeostasis in the human body. It is the constant bone remodeling throughout one's life that removes the old and damaged bone, keeping the skeletal system healthy. During the recent decade, many studies have demonstrated mechanisms for how osteoclastic bone resorption contributes to the subsequent bone formation in bone remodeling (Figure 2) and provided a wellrounded understanding of the roles of OCs in maintaining proper bone remodeling. 
Osteoporosis, the most prevalent disorder of bone remodeling by far, is characterized by the heightened activity of OCs $[6,7]$. Currently, the available treatments of osteoporosis comprise antiresorptive agents, such as bisphosphonate and denosumab, and anabolic treatments such as PTH $[6,13]$. However, most antiresorptive agents that suppress OC differentiation will concomitantly impair bone formation because of the coupling effect, leading to an unsatisfactory long-term effect and potentially increasing the likelihood of long-term adverse events, such as osteonecrosis of the jaw [120]. Thus, new agents under development for osteoporosis may try to retain the OC coupling factors while inhibiting OC functions. Odanacatib, a small-molecule inhibitor of CTSK, can decrease bone resorption without affecting OBs and appears to promote bone formation [106, 121, 122], probably because of the suppression on OC activity rather than the inhibition on OC viability, thus allowing continuous crosstalk between OCs and OBs. Unfortunately, because of the unforeseen cerebrovascular events, the clinical development of odanacatib was terminated. The side effects may result from the off-target effects of CTSK inhibitors on other members of the cathepsin family, such as cathepsins B, L, and S. Nonetheless, the experience learned from the underlying biology of CTSK inhibitors could guide future therapeutic approaches for osteoporosis: dissociating the inhibition of bone resorption from the coupled reduction in bone formation. This may be a promising strategy in the development of a new drug and we believe that a better outcome will be achieved when proper strategies are made to coordinate OCs and OBs in managing bone remodeling disorders.

\section{Conflicts of Interest}

All authors declare that there are no conflicts of interest.

\section{References}

[1] T. J. Martin and E. Seeman, "Bone remodelling: its local regulation and the emergence of bone fragility," Best Practice \& Research Clinical Endocrinology \& Metabolism, vol. 22, no. 5, pp. 701-722, 2008.

[2] D. J. Hadjidakis and I. I. Androulakis, "Bone remodeling," Annals of the New York Academy of Sciences, vol. 1092, no. 1, pp. 385-396, 2006.

[3] B.-J. Kim and J.-M. Koh, "Coupling factors involved in preserving bone balance," Cellular and Molecular Life Sciences, vol. 76, no. 7, pp. 1243-1253, 2019.

[4] C. Zuo, Y. Huang, R. Bajis et al., "Osteoblastogenesis regulation signals in bone remodeling," Osteoporosis International, vol. 23, no. 6, pp. 1653-1663, 2012.

[5] S. L. Teitelbaum, "Bone resorption by osteoclasts," Science, vol. 289, no. 5484, pp. 1504-1508, 2000.

[6] T. D. Rachner, S. Khosla, and L. C. Hofbauer, "Osteoporosis: now and the future," The Lancet, vol. 377, no. 9773, pp. 1276-1287, 2011.

[7] X. Feng and J. M. McDonald, "Disorders of bone remodeling," Annual Review of Pathology: Mechanisms of Disease, vol. 6, no. 1, pp. 121-145, 2011.

[8] N. C. Walsh and E. M. Gravallese, "Bone remodeling in rheumatic disease: a question of balance," Immunological Reviews, vol. 233, no. 1, pp. 301-312, 2010.
[9] H. Razi, A. I. Birkhold, R. Weinkamer, G. N. Duda, B. M. Willie, and S. Checa, "Aging leads to a dysregulation in mechanically driven bone formation and resorption," Journal of Bone and Mineral Research, vol. 30, no. 10, pp. 1864-1873, 2015.

[10] C. M. Karner and F. Long, "Glucose metabolism in bone," Bone, vol. 115, pp. 2-7, 2018.

[11] J. E. Compston, M. R. McClung, and W. D. Leslie, "Osteoporosis," The Lancet, vol. 393, no. 10169, pp. 364-376, 2019.

[12] J. N. Farr, M. Xu, M. M. Weivoda et al., "Targeting cellular senescence prevents age-related bone loss in mice," Nature Medicine, vol. 23, no. 9, pp. 1072-1079, 2017.

[13] T. Harsløf and B. L. Langdahl, "New horizons in osteoporosis therapies," Current Opinion in Pharmacology, vol. 28, pp. 38-42, 2016.

[14] J. Pajarinen, T. Lin, E. Gibon et al., "Mesenchymal stem cellmacrophage crosstalk and bone healing," Biomaterials, vol. 196, pp. 80-89, 2019.

[15] L. Wang, X. You, S. Lotinun, L. Zhang, N. Wu, and W. Zou, "Mechanical sensing protein PIEZO1 regulates bone homeostasis via osteoblast-osteoclast crosstalk," Nature Communications, vol. 11, no. 1, p. 282, 2020.

[16] A. Terashima and H. Takayanagi, "Overview of osteoimmunology," Calcified Tissue International, vol. 102, no. 5, pp. 503-511, 2018.

[17] K. Henriksen, M. A. Karsdal, and T. John Martin, "Osteoclast-derived coupling factors in bone remodeling," Calcified Tissue International, vol. 94, no. 1, pp. 88-97, 2014.

[18] A.-L. Gamblin, M. A. Brennan, A. Renaud et al., "Bone tissue formation with human mesenchymal stem cells and biphasic calcium phosphate ceramics: the local implication of osteoclasts and macrophages," Biomaterials, vol. 35, no. 36, pp. 9660-9667, 2014.

[19] Y. Han, X. You, W. Xing, Z. Zhang, and W. Zou, "Paracrine and endocrine actions of bone-the functions of secretory proteins from osteoblasts, osteocytes, and osteoclasts," Bone Research, vol. 6, p. 16, 2018.

[20] J. F. Charles and A. O. Aliprantis, "Osteoclasts: more than "bone eaters"," Trends in Molecular Medicine, vol. 20, no. 8, pp. 449-459, 2014.

[21] M. B. Madel, L. Ibanez, A. Wakkach et al., "Immune function and diversity of osteoclasts in normal and pathological conditions," Frontiers in Immunology, vol. 10, p. 1408, 2019.

[22] P.-L. Chung, S. Zhou, B. Eslami, L. Shen, M. S. LeBoff, and J. Glowacki, "Effect of age on regulation of human osteoclast differentiation," Journal of Cellular Biochemistry, vol. 115, no. 8, pp. 1412-1419, 2014.

[23] S. L. Perkins, R. Gibbons, S. Kling, and A. J. Kahn, “Age-related bone loss in mice is associated with an increased osteoclast progenitor pool," Bone, vol. 15, no. 1, pp. 65-72, 1994.

[24] J. A. Fafián-Labora, M. Morente-López, and M. C. Arufe, "Effect of aging on behaviour of mesenchymal stem cells," World Journal of Stem Cells, vol. 11, no. 6, pp. 337-346, 2019.

[25] U. Lindner, J. Kramer, J. Rohwedel, and P. Schlenke, "Mesenchymal stem or stromal cells: toward a better understanding of their biology?" Transfusion Medicine and Hemotherapy, vol. 37, no. 2, pp. 75-83, 2010.

[26] S. Khosla, "Odanacatib: location and timing are everything," Journal of Bone and Mineral Research, vol. 27, no. 3, pp. 506-508, 2012.

[27] K. W. Lyles, C. S. Colón-Emeric, J. S. Magaziner et al., "Zoledronic acid and clinical fractures and mortality after hip fracture," New England Journal of Medicine, vol. 357, no. 18, pp. 1799-1809, 2007. 
[28] B. Langdahl, S. Ferrari, and D. W. Dempster, "Bone modeling and remodeling: potential as therapeutic targets for the treatment of osteoporosis," Therapeutic Advances in Musculoskeletal Disease, vol. 8, no. 6, pp. 225-235, 2016.

[29] S. C. Manolagas, "Birth and death of bone cells: basic regulatory mechanisms and implications for the pathogenesis and treatment of osteoporosis," Endocrine Reviews, vol. 21, no. 2, pp. 115-137, 2000.

[30] N. A. Sims and T. J. Martin, "Coupling signals between the osteoclast and osteoblast: how are messages transmitted between these temporary visitors to the bone surface?" Frontiers in Endocrinology, vol. 6, p. 41, 2015.

[31] K. Matsuo and N. Irie, "Osteoclast-osteoblast communication," Archives of Biochemistry and Biophysics, vol. 473, no. 2, pp. 201-209, 2008.

[32] K. Henriksen, J. Bollerslev, V. Everts, and M. A. Karsdal, "Osteoclast activity and subtypes as a function of physiology and pathology-implications for future treatments of osteoporosis," Endocrine Reviews, vol. 32, no. 1, pp. 31-63, 2011.

[33] Z. Bar-Shavit, "The osteoclast: a multinucleated, hematopoietic-origin, bone-resorbing osteoimmune cell," Journal of Cellular Biochemistry, vol. 102, no. 5, pp. 1130-1139, 2007.

[34] Y. Guo, C. Xie, X. Li et al., "Succinate and its G-proteincoupled receptor stimulates osteoclastogenesis," Nature Communications, vol. 8, p. 15621, 2017.

[35] G. Stenbeck, "Formation and function of the ruffled border in osteoclasts," Seminars in Cell \& Developmental Biology, vol. 13, no. 4, pp. 285-292, 2002.

[36] D. J. Mellis, C. Itzstein, M. H. Helfrich, and J. C. Crockett, "The skeleton: a multi-functional complex organ. The role of key signalling pathways in osteoclast differentiation and in bone resorption," Journal of Endocrinology, vol. 211, no. 2, pp. 131-143, 2011.

[37] W. J. Boyle, W. S. Simonet, and D. L. Lacey, "Osteoclast differentiation and activation," Nature, vol. 423, no. 6937, pp. 337-342, 2003.

[38] H. Kajiya, "Calcium signaling in osteoclast differentiation and bone resorption," Advances in Experimental Medicine and Biology, vol. 740, pp. 917-932, 2012.

[39] S.-Y. Hwang and J. W. Putney Jr., "Calcium signaling in osteoclasts," Biochimica et Biophysica Acta (BBA)-Molecular Cell Research, vol. 1813, no. 5, pp. 979-983, 2011.

[40] S. A. Arkett, S. J. Dixon, and S. M. Sims, "Substrate influences rat osteoclast morphology and expression of potassium conductances," The Journal of Physiology, vol. 458, no. 1, pp. 633-653, 1992.

[41] H. Kajiya, F. Okamoto, H. Fukushima, K. Takada, and K. Okabe, "Mechanism and role of high-potassium-induced reduction of intracellular $\mathrm{Ca}^{2+}$ concentration in rat osteoclasts," American Journal of Physiology-Cell Physiology, vol. 285, no. 2, pp. C457-C466, 2003.

[42] J. H. Park, N. K. Lee, and S. Y. Lee, "Current understanding of RANK signaling in osteoclast differentiation and maturation," Molecules and Cells, vol. 40, no. 10, pp. 706-713, 2017.

[43] H. Bi, X. Chen, S. Gao et al., "Key triggers of osteoclastrelated diseases and available strategies for targeted therapies: a review," Frontiers in Medicine, vol. 4, p. 234, 2017.

[44] A. Mocsai, M. B. Humphrey, J. A. G. Van Ziffle et al., "The immunomodulatory adapter proteins DAP12 and Fc receptor-chain $(\mathrm{FcR})$ regulate development of functional osteoclasts through the Syk tyrosine kinase," Proceedings of the National Academy of Sciences, vol. 101, no. 16, pp. 6158-6163, 2004.
[45] H. Kanzaki, F. Shinohara, M. Kajiya, and T. Kodama, "The Keap1/Nrf2 protein axis plays a role in osteoclast differentiation by regulating intracellular reactive oxygen species signaling," Journal of Biological Chemistry, vol. 288, no. 32, pp. 23009-23020, 2013.

[46] D. V. Novack and S. L. Teitelbaum, "The osteoclast: friend or foe?" Annual Review of Pathology: Mechanisms of Disease, vol. 3, no. 1, pp. 457-484, 2008.

[47] D. A. Callaway and J. X. Jiang, "Reactive oxygen species and oxidative stress in osteoclastogenesis, skeletal aging and bone diseases," Journal of Bone and Mineral Metabolism, vol. 33, no. 4, pp. 359-370, 2015.

[48] K. Weiskopf, P. J. Schnorr, W. W. Pang et al., "Myeloid cell origins, differentiation, and clinical implications," Microbiology Spectrum, vol. 4, no. 5, 2016.

[49] N. Udagawa, N. Takahashi, T. Akatsu et al., "Origin of osteoclasts: mature monocytes and macrophages are capable of differentiating into osteoclasts under a suitable microenvironment prepared by bone marrow-derived stromal cells," Proceedings of the National Academy of Sciences, vol. 87, no. 18, pp. 7260-7264, 1990.

[50] R. Gruber, "Osteoimmunology: inflammatory osteolysis and regeneration of the alveolar bone," Journal of Clinical Periodontology, vol. 46, no. 21, pp. 52-69, 2019.

[51] M. Ponzetti and N. Rucci, "Updates on osteoimmunology: what's new on the cross-talk between bone and immune system," Frontiers in Endocrinology, vol. 10, p. 236, 2019.

[52] J. J. Cao, T. J. Wronski, U. Iwaniec et al., "Aging increases stromal/osteoblastic cell-induced osteoclastogenesis and alters the osteoclast precursor pool in the mouse," Journal of Bone and Mineral Research, vol. 20, no. 9, pp. 1659-1668, 2005.

[53] L. Kreja, R. E. Brenner, A. Tautzenberger et al., "Nonresorbing osteoclasts induce migration and osteogenic differentiation of mesenchymal stem cells," Journal of Cellular Biochemistry, vol. 109, no. 2, pp. 347-355, 2009.

[54] M. A. Karsdal, A. V. Neutzsky-Wulff, M. H. Dziegiel, C. Christiansen, and K. Henriksen, "Osteoclasts secrete nonbone derived signals that induce bone formation," Biochemical and Biophysical Research Communications, vol. 366, no. 2, pp. 483-488, 2008.

[55] K. Henriksen, K. V. Andreassen, C. S. Thudium et al., "A specific subtype of osteoclasts secretes factors inducing nodule formation by osteoblasts," Bone, vol. 51, no. 3, pp. 353-361, 2012.

[56] B.-J. Kim, Y.-S. Lee, S.-Y. Lee et al., "Afamin secreted from nonresorbing osteoclasts acts as a chemokine for preosteoblasts via the Akt-signaling pathway," Bone, vol. 51, no. 3, pp. 431-440, 2012.

[57] C. S. Thudium, I. Moscatelli, C. Flores et al., "A comparison of osteoclast-rich and osteoclast-poor osteopetrosis in adult mice sheds light on the role of the osteoclast in coupling bone resorption and bone formation," Calcified Tissue International, vol. 95, no. 1, pp. 83-93, 2014.

[58] T. Bellido, "Osteocyte-driven bone remodeling," Calcified Tissue International, vol. 94, no. 1, pp. 25-34, 2014.

[59] T. J. Heino, K. Kurata, H. Higaki, and H. K. Väänänen, "Evidence for the role of osteocytes in the initiation of targeted remodeling," Technology and Health Care, vol. 17, no. 1, pp. 49-56, 2009.

[60] S. R. Goldring, "The osteocyte: key player in regulating bone turnover," RMD Open, vol. 1, no. Suppl 1, Article ID e000049, 2015. 
[61] L. I. Plotkin, "Apoptotic osteocytes and the control of targeted bone resorption," Current Osteoporosis Reports, vol. 12, no. 1, pp. 121-126, 2014.

[62] O. Verborgt, G. J. Gibson, and M. B. Schaffler, "Loss of osteocyte integrity in association with microdamage and bone remodeling after fatigue in vivo," Journal of Bone and Mineral Research, vol. 15, no. 1, pp. 60-67, 2000.

[63] L. Cardoso, B. C. Herman, O. Verborgt, D. Laudier, R. J. Majeska, and M. B. Schaffler, "Osteocyte apoptosis controls activation of intracortical resorption in response to bone fatigue," Journal of Bone and Mineral Research, vol. 24, no. 4, pp. 597-605, 2009.

[64] T. J. Heino, T. A. Hentunen, and H. K. Väänänen, "Osteocytes inhibit osteoclastic bone resorption through transforming growth factor- $\beta$ : enhancement by estrogen*" Journal of Cellular Biochemistry, vol. 85, no. 1, pp. 185-197, 2002.

[65] T. Nakashima, M. Hayashi, T. Fukunaga et al., "Evidence for osteocyte regulation of bone homeostasis through RANKL expression," Nature Medicine, vol. 17, no. 10, pp. 1231-1234, 2011.

[66] J. Xiong, M. Onal, R. L. Jilka, R. S. Weinstein, S. C. Manolagas, and C. A. O'Brien, "Matrix-embedded cells control osteoclast formation," Nature Medicine, vol. 17, no. 10, pp. 1235-1241, 2011.

[67] J. Xiong, M. Piemontese, M. Onal et al., "Osteocytes, not osteoblasts or lining cells, are the main source of the RANKL required for osteoclast formation in remodeling bone," PLoS One, vol. 10, no. 9, Article ID e0138189, 2015.

[68] J. L. Crane and X. Cao, "Bone marrow mesenchymal stem cells and TGF- $\beta$ signaling in bone remodeling," Journal of Clinical Investigation, vol. 124, no. 2, pp. 466-472, 2014.

[69] L. Xian, X. Wu, L. Pang et al., "Matrix IGF-1 maintains bone mass by activation of mTOR in mesenchymal stem cells," Nature Medicine, vol. 18, no. 7, pp. 1095-1101, 2012.

[70] J. L. Crane and X. Cao, "Function of matrix IGF-1 in coupling bone resorption and formation," Journal of Molecular Medicine, vol. 92, no. 2, pp. 107-115, 2014.

[71] C. Sobacchi, E. Palagano, A. Villa, and C. Menale, "Soluble factors on stage to direct mesenchymal stem cells fate," Frontiers in Bioengineering and Biotechnology, vol. 5, p. 32, 2017.

[72] J. Kobolak, A. Dinnyes, A. Memic, A. Khademhosseini, and A. Mobasheri, "Mesenchymal stem cells: identification, phenotypic characterization, biological properties and potential for regenerative medicine through biomaterial microengineering of their niche," Methods, vol. 99, pp. 62-68, 2016.

[73] M. F. Pittenger, A. M. Mackay, S. C. Beck et al., "Multilineage potential of adult human mesenchymal stem cells," Science, vol. 284, no. 5411, pp. 143-147, 1999.

[74] B. Sacchetti, A. Funari, S. Michienzi et al., "Self-renewing osteoprogenitors in bone marrow sinusoids can organize a hematopoietic microenvironment," Cell, vol. 131, no. 2, pp. 324-336, 2007.

[75] A. I. Caplan, "New MSC: MSCs as pericytes are Sentinels and gatekeepers," Journal of Orthopaedic Research, vol. 35, no. 6, pp. 1151-1159, 2017.

[76] P. Su, Y. Tian, C. Yang et al., "Mesenchymal stem cell migration during bone formation and bone diseases therapy," International Journal of Molecular Sciences, vol. 19, no. 8, 2018.

[77] L. D. Carbonare, G. Innamorati, and M. T. Valenti, "Transcription factor Runx2 and its application to bone tissue engineering," Stem Cell Reviews and Reports, vol. 8, no. 3, pp. 891-897, 2012.

[78] W. Zhang, C. Zhu, Y. Wu et al., "VEGF and BMP-2 promote bone regeneration by facilitating bone marrow stem cell homing and differentiation," European Cells and Materials, vol. 27, pp. 1-12, 2014.

[79] A. Youssef, D. Aboalola, and V. K. Han, "The roles of insulinlike growth factors in mesenchymal stem cell niche," Stem Cells International, vol. 2017, Article ID 9453108, pp. 8-12, 2017.

[80] Y. Li, X. Yu, S. Lin, X. Li, S. Zhang, and Y.-H. Song, "Insulinlike growth factor 1 enhances the migratory capacity of mesenchymal stem cells," Biochemical and Biophysical Research Communications, vol. 356, no. 3, pp. 780-784, 2007.

[81] M. J. Dubon, J. Yu, S. Choi, and K.-S. Park, "Transforming growth factor $\beta$ induces bone marrow mesenchymal stem cell migration via noncanonical signals and N-cadherin," Journal of Cellular Physiology, vol. 233, no. 1, pp. 201-213, 2018.

[82] B. Awan, D. Turkov, C. Schumacher et al., "FGF2 induces migration of human bone marrow stromal cells by increasing core fucosylations on N-glycans of integrins," Stem Cell Reports, vol. 11, no. 2, pp. 325-333, 2018.

[83] A. Li, X. Xia, J. Yeh et al., "PDGF-AA promotes osteogenic differentiation and migration of mesenchymal stem cell by down-regulating PDGFRalpha and derepressing BMPSmad1/5/8 signaling," PLoS One, vol. 9, no. 12, Article ID e113785, 2014.

[84] S. Salha, S. Gehmert, V. Brebant et al., "PDGF regulated migration of mesenchymal stem cells towards malignancy acts via the PI3K signaling pathway," Clinical Hemorheology and Microcirculation, vol. 70, no. 4, pp. 543-551, 2019.

[85] R. O. C. Oreffo, G. R. Mundy, S. M. Seyedin, and L. F. Bonewald, "Activation of the bone-derived latent TGF beta complex by isolated osteoclasts," Biochemical and Biophysical Research Communications, vol. 158, no. 3, pp. 817-823, 1989.

[86] B. Wildemann, A. Kadow-Romacker, N. P. Haas, and G. Schmidmaier, "Quantification of various growth factors in different demineralized bone matrix preparations," Journal of Biomedical Materials Research Part A, vol. 81A, no. 2, pp. 437-442, 2007.

[87] G. A. Howard, B. L. Bottemiller, R. T. Turner, J. I. Rader, and D. J. Baylink, "Parathyroid hormone stimulates bone formation and resorption in organ culture: evidence for a coupling mechanism," Proceedings of the National Academy of Sciences, vol. 78, no. 5, pp. 3204-3208, 1981.

[88] Y. Tang, X. Wu, W. Lei et al., "TGF- $\beta 1$-induced migration of bone mesenchymal stem cells couples bone resorption with formation," Nature Medicine, vol. 15, no. 7, pp. 757-765, 2009.

[89] M. Centrella and E. Canalis, "Local regulators of skeletal growth: a perspective*” Endocrine Reviews, vol. 6, no. 4, pp. 544-551, 1985.

[90] H. Hanamura, Y. Higuchi, M. Nakagawa, H. Iwata, H. Nogami, and M. R. Urist, "Solubilized bone morphogenetic protein (BMP) from mouse osteosarcoma and rat demineralized bone matrix," Clinical Orthopaedics and Related Research, vol. 148, pp. 281-290, 1980.

[91] U. Mayr-Wohlfart, J. Waltenberger, H. Hausser et al., "Vascular endothelial growth factor stimulates chemotactic migration of primary human osteoblasts," Bone, vol. 30, no. 3, pp. 472-477, 2002. 
[92] E. Mihara, H. Hirai, H. Yamamoto et al., "Active and watersoluble form of lipidated Wnt protein is maintained by a serum glycoprotein afamin/alpha-albumin," Elife, vol. 5, 2016.

[93] S. Vukicevic and L. Grgurevic, "BMP-6 and mesenchymal stem cell differentiation," Cytokine \& Growth Factor Reviews, vol. 20, no. 5-6-6, pp. 441-448, 2009.

[94] R. Garimella, S. E. Tague, J. Zhang et al., "Expression and synthesis of bone morphogenetic proteins by osteoclasts: a possible path to anabolic bone remodeling," Journal of Histochemistry \& Cytochemistry, vol. 56, no. 6, pp. 569-577, 2008.

[95] K. Matsuoka, K.-a. Park, M. Ito, K. Ikeda, and S. Takeshita, "Osteoclast-derived complement component 3a stimulates osteoblast differentiation," Journal of Bone and Mineral Research, vol. 29, no. 7, pp. 1522-1530, 2014.

[96] E. C. Walker, N. E. McGregor, I. J. Poulton et al., "Cardiotrophin-1 is an osteoclast-derived stimulus of bone formation required for normal bone remodeling," Journal of Bone and Mineral Research, vol. 23, no. 12, pp. 2025-2032, 2008.

[97] S. Takeshita, T. Fumoto, K. Matsuoka et al., "Osteoclastsecreted CTHRC1 in the coupling of bone resorption to formation," Journal of Clinical Investigation, vol. 123, no. 9, pp. 3914-3924, 2013.

[98] K. Ota, P. Quint, M. M. Weivoda et al., "Transforming growth factor beta 1 induces CXCL16 and leukemia inhibitory factor expression in osteoclasts to modulate migration of osteoblast progenitors," Bone, vol. 57, no. 1, pp. $68-75,2013$.

[99] M. Grano, F. Galimi, G. Zambonin et al., "Hepatocyte growth factor is a coupling factor for osteoclasts and osteoblasts in vitro," Proceedings of the National Academy of Sciences, vol. 93, no. 15, pp. 7644-7648, 1996.

[100] H. T. Chen, H. K. Tsou, C. H. Chang, and C. H. Tang, "Hepatocyte growth factor increases osteopontin expression in human osteoblasts through PI3K, Akt, c-Src, and AP-1 signaling pathway," PLoS One, vol. 7, no. 6, Article ID e38378, 2012.

[101] H. Xie, Z. Cui, L. Wang et al., "PDGF-BB secreted by preosteoclasts induces angiogenesis during coupling with osteogenesis," Nature Medicine, vol. 20, no. 11, pp. 12701278, 2014.

[102] M. A. Sanchez-Fernandez, A. Gallois, T. Riedl, P. Jurdic, and B. Hoflack, "Osteoclasts control osteoblast chemotaxis via PDGF-BB/PDGF receptor beta signaling," PLoS One, vol. 3, no. 10, p. e3537, 2008.

[103] K. Kubota, C. Sakikawa, M. Katsumata, T. Nakamura, and K. Wakabayashi, "Platelet-derived growth factor BB secreted from osteoclasts acts as an osteoblastogenesis inhibitory factor," Journal of Bone and Mineral Research, vol. 17, no. 2, pp. 257-265, 2002.

[104] L. Pederson, M. Ruan, J. J. Westendorf, S. Khosla, and M. J. Oursler, "Regulation of bone formation by osteoclasts involves Wnt/BMP signaling and the chemokine sphingosine-1-phosphate," Proceedings of the National Academy of Sciences, vol. 105, no. 52, pp. 20764-20769, 2008.

[105] S. Weske, M. Vaidya, A. Reese et al., "Targeting sphingosine1-phosphate lyase as an anabolic therapy for bone loss," Nature Medicine, vol. 24, no. 5, pp. 667-678, 2018.

[106] S. Lotinun, R. Kiviranta, T. Matsubara et al., "Osteoclastspecific cathepsin K deletion stimulates S1P-dependent bone formation," Journal of Clinical Investigation, vol. 123, no. 2, pp. 666-681, 2013.
[107] B.-J. Kim, Y.-S. Lee, S.-Y. Lee et al., "Osteoclast-secreted SLIT3 coordinates bone resorption and formation," Journal of Clinical Investigation, vol. 128, no. 4, pp. 1429-1441, 2018.

[108] N. Z. Angel, N. Walsh, M. R. Forwood, M. C. Ostrowski, A. I. Cassady, and D. A. Hume, "Transgenic mice overexpressing tartrate-resistant acid phosphatase exhibit an increased rate of bone turnover," Journal of Bone and Mineral Research, vol. 15, no. 1, pp. 103-110, 2000.

[109] A. R. Hayman and T. M. Cox, "Tartrate-resistant acid phosphatase knockout mice," Journal of Bone and Mineral Research, vol. 18, no. 10, pp. 1905-1907, 2003.

[110] Y. Ikebuchi, S. Aoki, M. Honma et al., "Coupling of bone resorption and formation by RANKL reverse signalling," Nature, vol. 561, no. 7722, pp. 195-200, 2018.

[111] T. Negishi-Koga, M. Shinohara, N. Komatsu et al., "Suppression of bone formation by osteoclastic expression of semaphorin 4D," Nature Medicine, vol. 17, no. 11, pp. 1473-1480, 2011.

[112] K. Ota, P. Quint, M. Ruan et al., "Sclerostin is expressed in osteoclasts from aged mice and reduces osteoclast-mediated stimulation of mineralization," Journal of Cellular Biochemistry, vol. 114, no. 8, pp. 1901-1907, 2013.

[113] D. Li, J. Liu, B. Guo et al., "Osteoclast-derived exosomal miR214-3p inhibits osteoblastic bone formation," Nature Communications, vol. 7, p. 10872, 2016.

[114] F. L. Yuan, Q. Y. Wu, Z. N. Miao et al., "Osteoclast-derived extracellular vesicles: novel regulators of osteoclastogenesis and osteoclast-osteoblasts communication in bone remodeling," Frontiers in Physiology, vol. 9, p. 628, 2018.

[115] T. L. Andersen, M. E. Abdelgawad, H. B. Kristensen et al., "Understanding coupling between bone resorption and formation," The American Journal of Pathology, vol. 183, no. 1, pp. 235-246, 2013.

[116] M. Furuya, J. Kikuta, S. Fujimori et al., "Direct cell-cell contact between mature osteoblasts and osteoclasts dynamically controls their functions in vivo," Nature Communications, vol. 9, no. 1, p. 300, 2018.

[117] N. W. Gale, S. J. Holland, D. M. Valenzuela et al., "Eph receptors and ligands comprise two major specificity subclasses and are reciprocally compartmentalized during embryogenesis," Neuron, vol. 17, no. 1, pp. 9-19, 1996.

[118] E. B. Pasquale, "Eph receptor signalling casts a wide net on cell behaviour," Nature Reviews Molecular Cell Biology, vol. 6, no. 6, pp. 462-475, 2005.

[119] C. Zhao, N. Irie, Y. Takada et al., "Bidirectional ephrinB2EphB4 signaling controls bone homeostasis," Cell Metabolism, vol. 4, no. 2, pp. 111-121, 2006.

[120] C. Reyes, M. Hitz, D. Prieto-Alhambra, and B. Abrahamsen, "Risks and benefits of bisphosphonate therapies," Journal of Cellular Biochemistry, vol. 117, no. 1, pp. 20-28, 2016.

[121] M. T. Drake, B. L. Clarke, M. J. Oursler, and S. Khosla, "Cathepsin K inhibitors for osteoporosis: biology, potential clinical utility, and lessons learned," Endocrine Reviews, vol. 38, no. 4, pp. 325-350, 2017.

[122] T. Cusick, C. M. Chen, B. L. Pennypacker et al., "Odanacatib treatment increases hip bone mass and cortical thickness by preserving endocortical bone formation and stimulating periosteal bone formation in the ovariectomized adult rhesus monkey," Journal of Bone and Mineral Research, vol. 27, no. 3, pp. 524-537, 2012. 